\title{
Monte Carlo analysis of the spectral photon emission and extraction efficiency of organic light-emitting devices
}

\author{
Aldo Badano a) and Jerzy Kanickib) \\ Solid-State Electronic Laboratory, Department of Electrical Engineering and Computer Science, \\ The University of Michigan, Ann Arbor, Michigan 48109
}

(Received 19 February 2001; accepted for publication 18 May 2001)

\begin{abstract}
We report on a Monte Carlo method for modeling light transport phenomena in multilayer organic polymer light-emitting devices on plastic flexible substrates. The method allows modeling of Cartesian geometrical structures describing the fate of photons through multiple scattering events determined by the wavelength-dependent material optical properties. We apply the method to analyze the wavelength distribution of emitted light spectra. We find that for all organic polymers considered, the light emission is slightly shifted toward the longer wavelengths, and that this shift is maximum for light emissions with peaks around $530 \mathrm{~nm}$. The photon extraction efficiency is higher $(0.430)$ for organic polymers emitting in the longer wavelengths, while the photon absorbed fraction is higher (0.676) for spectra with a maximum in the short wavelengths. (C) 2001 American
\end{abstract} Institute of Physics. [DOI: 10.1063/1.1385571]

\section{INTRODUCTION}

The increase in the luminous efficiency of organic lightemitting devices (OLEDs) remains the focus of many efforts centered mostly on controlling charge transport and increasing the photoluminescent efficiency of organic materials. Currently, the analysis of the optical transport processes in OLEDs has obtained much attention. It is known that only about one fifth of the light generated in an OLED is emitted through the top surface of a simple OLED structure on a glass substrate. ${ }^{1}$ The optical losses are related to absorption in the organic material and edge emission of waveguided modes. For a typical organic semiconductor with refractive index of 2.0, the escape cone angle into a substrate with an index of 1.5 is $48.6^{\circ}$ which corresponds to a solid angle of only $\sim 6 \%$. Several attempts have been made to reduce the waveguided light fraction by using structured surfaces $^{2-4}$ and films, ${ }^{5}$ and spherical scatterers within the organic film. ${ }^{6}$ In addition to affecting the efficiency of the OLED, photon transport processes influence the spectral and angular distributions of the emitted light intensity. Both distributions are important design parameters for achieving full-color efficient OLEDs for flat-panel display applications.

After the simple analysis of OLED efficiency presented in Ref. 1, several groups have investigated the effect of light transport in multilayer structures. ${ }^{3,7-9}$ The method we present in this article is based on a Monte Carlo (MC) approach. ${ }^{10,11}$

\section{SIMULATION METHOD}

The MC method makes use of the generation of photons with random direction according to a distribution function describing the nature of the light emission. In this analysis,

\footnotetext{
${ }^{a}$ Current address: U.S. Food and Drug Administration, Center for Devices and Radiological Health, 12720 Twinbrook Parkway, Rockville, MD 20857; Electronic mail: agb@cdrh.fda.gov

b)Electronic mail: kanicki@eecs.umich.edu
}

the light source within the organic polymer layer is considered isotropic from a single point situated in the center of the device (see Fig. 1). To obtain an isotropic distribution of the directional cosines, we sample the three directional cosines that define the photon direction according to

$$
\begin{aligned}
& C_{x}=\sqrt{1-\xi_{1}^{2}} \sin 2 \pi \xi_{2}, \\
& C_{y}=\sqrt{1-\xi_{1}^{2}} \cos 2 \pi \xi_{2}, \\
& C_{z}=\xi_{2},
\end{aligned}
$$

where $\xi_{1}$ and $\xi_{2}$ are uniformly sampled in $[0,1)$. The energy of the photon source is defined by a table that corresponds to a specific spectral light emission. The initial photon polarization vector is sampled uniformly in the $4 \pi$ space, therefore assuming unpolarized light emission. The photon histories are then followed through a sequence of interactions that includes absorption and Fresnel refraction. A unique advantage of this simulation method is its ability to model bulk absorption events, thin-film coatings and rough surfaces, while keeping track of the photon polarization state. Bulk absorption is determined by sampling the probability of a photon being absorbed after a path of length $l$ by the exponential law

$$
P(l)=1-\mathrm{e}^{-\mu_{a b}(\lambda) l},
$$

where $\mu_{a b}(\lambda)$ is the wavelength-dependent linear absorption coefficient. At the optical boundaries, an analysis is performed depending on the surface type and material properties using Fresnel's equations and considering the polarization of the incoming photon. ${ }^{12}$ When the film thickness is comparable to the photon wavelength, we use modified Fresnel coefficients to describe the interference effects of optically thin films. The reflection and transmission coefficients are then interpreted as probabilities. The simulation outcome is calculated by a statistical average of the fate of all histories according to the desired quantity to be evaluated 


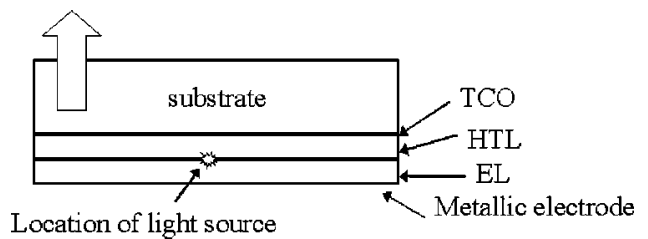

FIG. 1. Schematic cross section of the OLED structure used for the Monte Carlo simulations, showing the location of the centered point light source at the interface between the hole-transporting and emissive organic polymer layers.

for each experiment. Possible reporting options include the angular and spectral distributions of the emitted photons, the point-spread function, the specular and diffuse reflection coefficients, and a summary of scattering event statistics.

From the light emission at the luminescent center until the photons emerge, multiple scattering events take place within the multilayer OLED structure. For the purpose of our analysis, we define the device external quantum efficiency as

$$
\eta_{E}=\eta_{\text {in }} \eta_{p e}
$$

where $\eta_{i n}$ is the intrinsic quantum efficiency related to carrier recombination and photoluminescent fraction, and $\eta_{p e}$ is the photon extraction efficiency. We introduce $\eta_{p e}$ to represent the probability that a photon generated at the luminescent center within the OLED, emerges through the front surface of the device (through the transparent electrode), thereby contributing to luminance. The $\eta_{p e}$ depends strongly on the device structure and on the material and surface properties, and is always less than unity due to light absorption, waveguiding, and edge emissions. We can summarize the relevant physical processes that occur as

$$
\eta_{p e}=1-\eta_{w a}-\eta_{a b}-\eta_{t r}
$$

where $\eta_{w a}$ is the fraction of photons that are waveguided within the structure and exit through the device edges, $\eta_{a b}$ is the absorbed fraction, and $\eta_{t r}$ is the fraction transmitted through the metallic electrode deposited in the side opposite the direction of the desired light emission. The top metallic cathode electrode of all the OLED structures modeled in this article is an aluminum layer deposited by vacuum evaporation. ${ }^{13}$ We considered $\eta_{t r}=0$ in all simulations presented in this article. In this work, we neglected the photoluminescence quenching due to polymer composition or blend variations and the presence of carrier flow within the OLED. Electric field induced photoluminescence (PL) quenching in conjugated polymers, which is caused by exciton dissociation, is well known, ${ }^{14}$ but cannot be implemented easily in this calculation. It should be noted that this PL quenching is not important at the OLED operating point.

\section{RESULTS AND DISCUSSION}

We measured the index of refraction, absorption, PL, and electro-luminescence (EL) of three organic polymers (A, B, and $\mathrm{C}$ ) with peak emissions in a different region of the visible spectrum. The refractive index (considered equal for the three organic polymers) and absorption characteristics are shown in Fig. 2. We used the PL spectrum as the photon

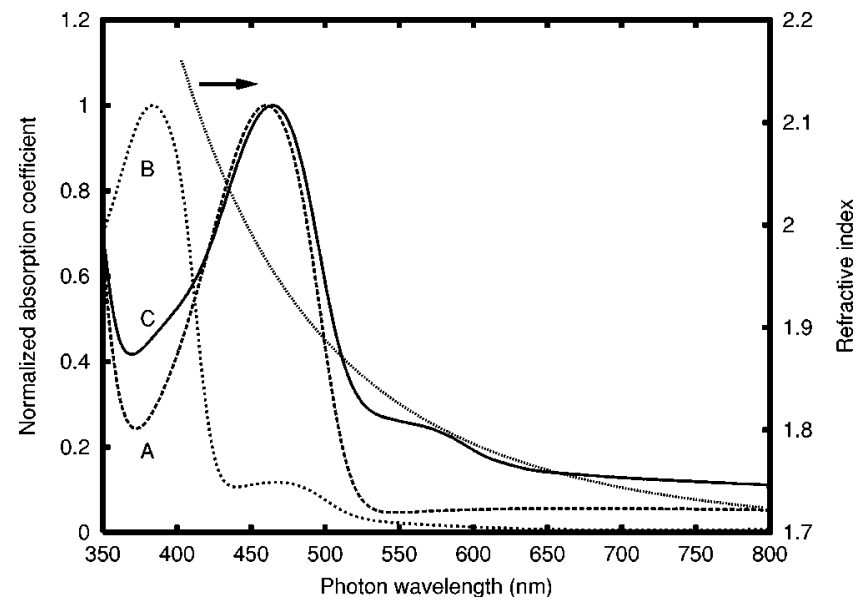

FIG. 2. Refractive index and normalized absorption coefficient of organic polymers $\mathrm{A}, \mathrm{B}$, and $\mathrm{C}$ modeled in this work.

energy distribution at the source for the MC histories. The OLED structure modeled in this article (see Fig. 1) is a heterostructure OLED described by $\mathrm{He}$ et al. ${ }^{13}$ as one with an aluminum cathode electrode and a transparent anode ITO electrode $(160 \mathrm{~nm}$, refractive index of 1.8). The organic polymer film thickness used was $200 \mathrm{~nm}$. The transparent substrate index of refraction was 1.5 and its thickness was $900 \mu \mathrm{m}$.

In Fig. 3, we present results of the simulated light (SL) emission wavelength distribution. For the three PL spectra considered, the measured EL spectrum is only slightly shifted toward the longer wavelengths. Our MC simulation results are consistent with this trend. By computing separately the simulated emission from a device structure having absorption in the organic polymer film, and from another with no absorption but with a thin-film transparent layer between the organic material and the substrate, we have confirmed that the decrease in power efficiency of the shorter wavelength range is associated with absorption in the organic material, while the increase in strength at longer wavelengths is caused by interference effects associated with the transparent conductive coating. Our simulation method can correctly predict the shift in maximum wavelength of each EL spectrum with respect to the PL spectrum of each material. It should be noticed that the maximum of the SL spectrum of each material is located very close to the corresponding maximum of the measured EL spectrum. However, especially in the longer wavelengths, a discrepancy exists between the EL and the MC calculated SL that cannot be explained in terms of optical transport phenomena. The discrepancy between the SL and EL suggests that the spectral light emission at the source might not be completely accurate. For our MC simulations, we have assumed that the spectrum at the source is equal to the PL spectrum measured for each polymer. The actual light emission in OLEDs could differ from that of the PL, reflecting the geometrical and structural differences in both the solid-state films in the OLED and in the device structure. Photoluminescence quenching due to the presence of carriers might also affect the spectral emission by modifying the ratio between radiative and nonradiative recombination. 

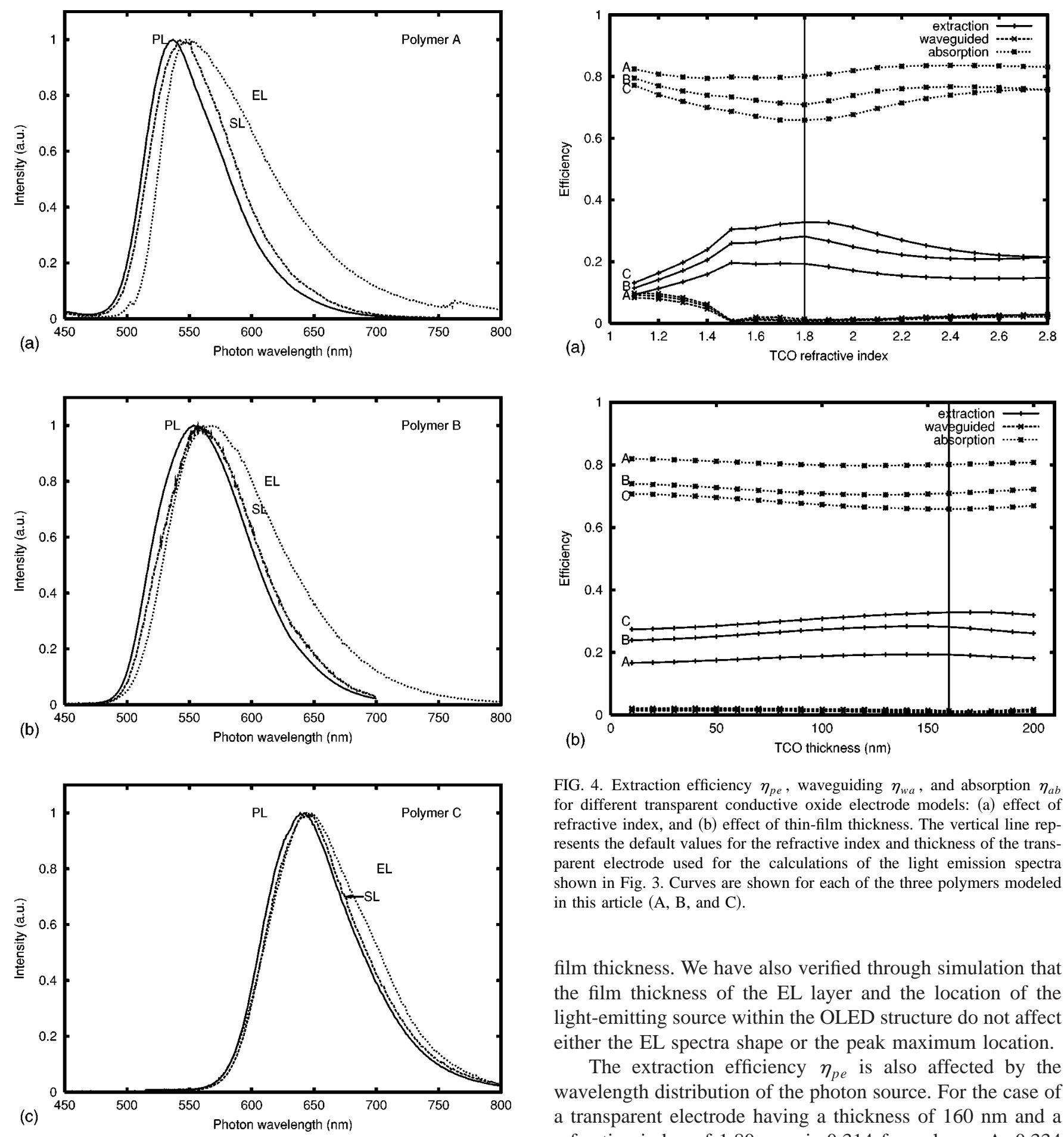

FIG. 4. Extraction efficiency $\eta_{p e}$, waveguiding $\eta_{w a}$, and absorption $\eta_{a b}$ for different transparent conductive oxide electrode models: (a) effect of refractive index, and (b) effect of thin-film thickness. The vertical line represents the default values for the refractive index and thickness of the transparent electrode used for the calculations of the light emission spectra shown in Fig. 3. Curves are shown for each of the three polymers modeled in this article (A, B, and $\mathrm{C})$.

film thickness. We have also verified through simulation that the film thickness of the EL layer and the location of the light-emitting source within the OLED structure do not affect either the EL spectra shape or the peak maximum location.

The extraction efficiency $\eta_{p e}$ is also affected by the wavelength distribution of the photon source. For the case of a transparent electrode having a thickness of $160 \mathrm{~nm}$ and a refractive index of $1.80, \eta_{p e}$ is 0.314 for polymer $\mathrm{A}, 0.334$ for polymer $\mathrm{B}$, and 0.430 for polymer $\mathrm{C}$. The absorbed fraction $\eta_{a b}$ is $0.676,0.655$, and 0.553 , and the wave-guided fraction $\eta_{w a}$ is $0.001,0.011$, and 0.017 , respectively, for polymers $\mathrm{A}, \mathrm{B}$, and $\mathrm{C}$. The low $\eta_{w a}$ is caused by high absorption in the organic film. In Fig. 4, we present results showing the change in the values of $\eta_{p e}, \eta_{a b}$, and $\eta_{w a}$ with the index of refraction (thickness) of the transparent conducting oxide (TCO) electrode covering the range from 1.1 to 2.7 $(10-200 \mathrm{~nm})$. We find that both the thickness and the refractive index of the transparent electrode have a minor effect on the shift of the SL spectrum with respect to the PL emission. We obtained a maximum $\eta_{p e}$ for a TCO thickness and a refractive index of about $170 \mathrm{~nm}$ and 1.85 , respectively. experimentally that the PL spectra shape is independent of 


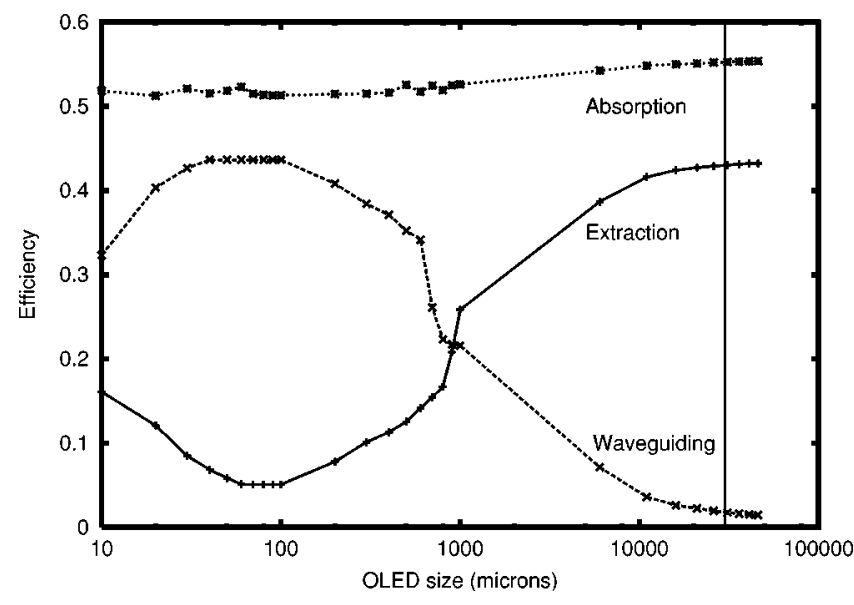

FIG. 5. Extraction efficiency $\eta_{p e}$, waveguiding $\eta_{w a}$, and absorption $\eta_{a b}$ as a function of OLED size for polymer B. Similar curves were obtained for polymers $\mathrm{A}$ and $\mathrm{C}$. The vertical line represents the default device size used throughout this article.

The waveguide modes are determined by the geometry of the OLED stack that defines a total internal reflection (TIR) angle $\left(\alpha_{\text {TIR }}\right)$ with respect to the device plane, beyond which all photons are emitted through the edges. Since our source of photons is isotropic, we expect lower $\eta_{w a}$ for larger devices due to (1) an increased probability of reflection and scattering going into the solid angle defined by $\alpha_{\text {TIR }}$, and (2) increased absorption in the organic polymer layer. The simulation result presented in Fig. 5 confirms this assumption. For OLED sizes larger than $50 \mu \mathrm{m}, \eta_{p e}$ steadily increases because more photons impinge directly (as a first interaction) into the substrate. For small device sizes below 2 $\mathrm{mm}$, the absorbed fraction $\eta_{a b}$ remains constant while the waveguiding decreases as the photon extraction efficiency increases. When the device size decreases below $100 \mu \mathrm{m}$, lateral boundary conditions become important and edge effects are observed. In our simulations, no additional structure is present at the edges of the device (i.e., the sides are considered to be smooth surfaces in contact with air). Photons reflected from the sides of the substrate at larger angles with respect to the surface normal have a larger probability of exiting the device through the top surface, thereby contributing to the extraction efficiency and to the luminance. For large device sizes, $\eta_{p e}$ and $\eta_{a b}$ increase while the waveguiding continues to decrease at a faster rate. The results also confirm that since most of the photon waveguiding occurs in the transparent substrate, the absorption in the organic polymer film has a minor effect on reducing the light edge emission and increasing the photon extraction efficiency.

\section{CONCLUSION}

In this article, we have presented a method to model light transport processes in OLEDs based on Monte Carlo techniques. For all organic polymers considered in this work, our results show that the simulated light emission is shifted toward the longer wavelengths, consistent with experimental measurements of EL. We also showed that the photon extraction efficiency is reduced by light absorption and waveguiding. Reduction of the OLED waveguided fraction can be obtained by using structured substrates or by tuning the refractive index of the layers to maximize the transmission and reduce the total internal reflection at each interface. Of particular importance to this approach is the interface between the organic polymers and the TCO/substrate, as well as details of the structure and index of refraction of the layers in plastic substrates. The presence of optimized thin-film coatings in a plastic substrate could contribute to a decrease in internally reflected trapped photons. A combination of these two approaches could result in optimal OLED structures with high photon extraction efficiency.

\section{ACKNOWLEDGMENTS}

The authors thank Y. He, Y. Hong, and S.-J. Lee for the measurements of optical material properties and PL and EL spectra. This research was supported in part by a U.S. Army postdoctoral fellowship No. DAMD17-00-1-0635 to one of the authors (A.B.).

${ }^{1}$ N. C. Greenham, R. H. Friend, and D. D. C. Bradley, Adv. Mater. 6, 491 (1994).

${ }^{2}$ I. Schnitzer, E. Yablonovitch, C. Caneau, T. J. Gmitter, and A. Sherer, Appl. Phys. Lett. 63, 2174 (1993).

${ }^{3}$ G. Gu, D. Z. Garbuzov, P. E. Burrows, S. Venkatesh, and S. R. Forrest, Opt. Lett. 22, 396 (1997).

${ }^{4}$ R. Windisch, P. Heremans, A. Knobloch, P. Kiesel, G. H. Döhler, B. Dutta, and G. Borghs, Appl. Phys. Lett. 74, 2256 (1999).

${ }^{5}$ J. M. Lupton, B. J. Matterson, I. D. W. Samuel, M. J. Jory, and W. L. Barnes, Appl. Phys. Lett. 77, 3340 (2000).

${ }^{6}$ T. Yamasaki, K. Sumioka, and T. Tsutsui, Appl. Phys. Lett. 76, 1243 (2000).

${ }^{7}$ F. Cacialli, S. E. Burns, and H. Becker, Opt. Mater. 9, 168 (1998).

${ }^{8}$ S. E. Burns, N. C. Greenham, and R. H. Friend, Synth. Met. 76, 205 (1996).

${ }^{9}$ V. Bulović, V. B. Khalfin, G. Gu, P. E. Burrows, D. Z. Garbuzov, and S. R. Forrest, Phys. Rev. B 58, 3730 (1998).

${ }^{10}$ A. Badano, Ph.D. thesis, University of Michigan, Ann Arbor, MI, 1999.

${ }^{11}$ G. F. Knoll and T. F. Knoll, IEEE Trans. Nucl. Sci. 35, 872 (1988).

${ }^{12} \mathrm{M}$. Born and E. Wolf, Principles of Optics, 3rd ed. (Pergamon, New York, 1965).

${ }^{13}$ Y. He, S. Gong, R. Hattori, and J. Kanicki, Appl. Phys. Lett. 74, 2265 (1999).

${ }^{14}$ M. Deussen, M. Sheidler, and H. Bassler, Synth. Met. 73, 123 (1995). 